\title{
On the relevance of magnetohydrodynamic pumping in solar coronal loop simulation experiments
}

\author{
J. Tenfelde, ${ }^{1}$ P. Kempkes,${ }^{2}$ F. Mackel, ${ }^{1}$ S. Ridder,${ }^{1}$ H. Stein, ${ }^{1}$ T. Tacke,${ }^{1}$ and $H$. \\ Soltwisch ${ }^{1}$ \\ 1)Institute of Experimental Physics V - Laser and Plasma Physics Group \\ Ruhr Universität Bochum, D-44780 Bochum, Germany \\ ${ }^{2)}$ Ernst-Moritz-Arndt-Universität Greifswald and \\ MPI for Plasma Physics, D-17491 Greifswald, Germany
}

A magnetohydrodynamic pumping mechanism was proposed by Bellan [Bellan, Phys. Plasmas 10, 1999 (2003)] to explain the formation of highly collimated plasmafilled magnetic flux tubes in certain solar coronal loop simulation experiments. In this paper, measurements on such an experiment are compared to the predictions of Bellan's pumping and collimation model. Significant discrepancies between theoretical implications and experimental observations have prompted more elaborate investigations by making use of pertinent modifications of the experimental device. On the basis of these studies it is concluded that the proposed MHD pumping mechanism does not play a crucial role for the formation and temporal evolution of the arched plasma structures that are generated in the coronal loop simulation experiments under consideration.

\section{INTRODUCTION}

Coronal loops protruding from the solar surface into the atmosphere are well-known phenomena, which have been the subject of numerous theoretical and numerical studies (see, e.g. Crooker et al. ${ }^{1}$, Török and Kliem ${ }^{2}$ and references therein). Commonly, these loops are supposed to be plasma-filled magnetic flux tubes, although the detailed mechanisms behind plasma filling, dynamic flows and eventual eruptions are still unclear. In order to provide a deeper insight into certain aspects of the observed behavior, Bellan and co-workers ${ }^{3,4}$ constructed a laboratory experiment which is able to produce magnetized plasma configurations similar to solar prominences under well controlled conditions.

In this experiment, arch-shaped plasmas were generated with the aid of a horseshoe magnet, whose poles protruded into a vacuum chamber and served at the same time as inlets for a hydrogen gas puff and as electrodes to drive a strong current along the field lines (for details see section III). Despite the bulged magnetic topology with field lines spread out at the apex of the arch and crowded together at the foot points, the resulting plasma-filled flux tubes were well collimated and had a remarkably uniform cross section along the distance between the electrodes. 
To explain this uniformity, Bellan ${ }^{5}$ proposed a magnetohydrodynamic (MHD) pumping mechanism due to an axial force which is brought about by the flared current profile. According to this model, the force effectively ingests plasma from the electrode gas orifices and then accelerates this plasma to flow as a jet into the magnetic flux tube. The flows from both electrodes convect frozen-in magnetic flux to the apex region where they stagnate, thus increasing the local azimuthal field and collimating the flux tube via the pinch effect.

Various experimental findings have been published by Bellan and co-workers to back up this model by checking consequences of the predicted plasma transport: Tripathi, Bellan and Yun ${ }^{6}$ claimed an increase of the plasma density in the apex region by many orders of magnitude over the background neutral gas density and reported flow velocity measurements of a few $\mathrm{cm} / \mu \mathrm{s}$ via spectral line Doppler shifts along the discharge arch. You, Yun and Bellan ${ }^{7,8}$ and Yun and Bellan ${ }^{9}$ published similar values for both the density increase and the typical flow velocities. The latter publications refer to a different type of plasma source which, however, had comparable properties with respect to the relevant plasma parameters and the bulged magnetic field structure.

In this paper we revisit the evidence for the MHD pumping mechanism in the coronal loop simulation experiment by presenting measurements and observations in our FlareLab ${ }^{10}$ device, which was designed in close similarity to the one operated by Bellan and co-workers. Substantial discrepancies with respect to the model predictions prompted us to redesign the plasma source such that the bulged field geometry of the horseshoe magnet was replaced by concentric circular field lines of a strong line current. Although this modification voided the proposed pumping mechanism, we observed very similar discharge evolutions in both configurations including the expansion behavior and the resulting plasma densities.

The following section will give a brief summary of the MHD pumping mechanism and the underlying assumptions. Next, our experimental setup will be described and compared to the device that was used by Tripathi et al. ${ }^{6}$ to corroborate the effectiveness of the pumping process. In section IV our experimental findings with respect to the collimation process and the density increase during the expansion phase will be discussed. In section $V$ the modification of the plasma source will be explained and it will be shown that it has little influence on the overall performance. Finally, our results and conclusions will be summarized in section VI.

\section{THE MAGNETOHYDRODYNAMIC PUMPING MECHANISM}

The MHD pumping and collimation model proposed by Bellan ${ }^{5}$ is based on the concept of frozen-in magnetic flux and refers to a simplified magnetic field geometry as sketched in Fig. 1. Instead of a loop, the initial configuration is assumed to be a straight tube reminiscent of a magnetic bottle, which contains plasma particles 


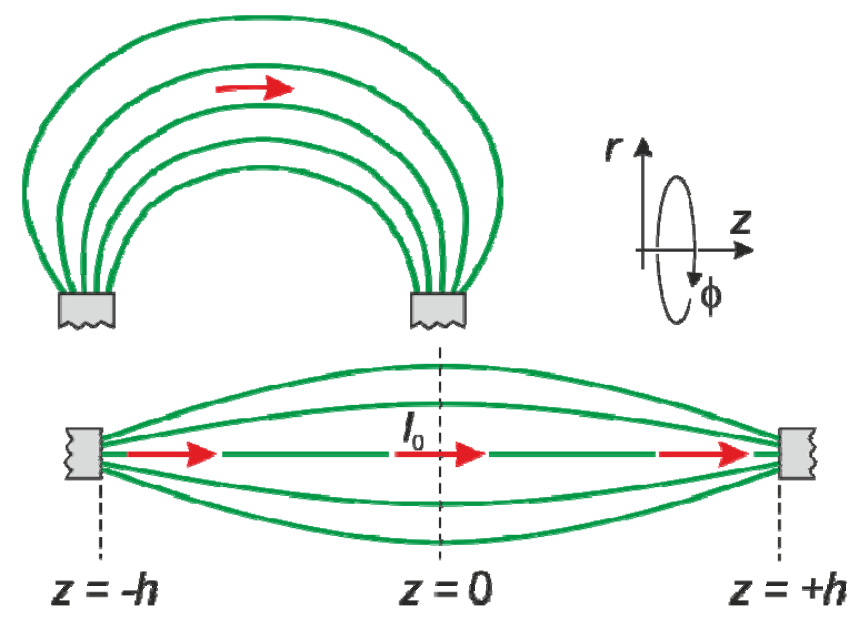

FIG. 1. (Color online) Flux tube geometry used in the model and relation to the arched field of a horseshoe magnet as applied in the experiment.

mainly near the foot points. By driving a current $I_{0}$ along the axis the field lines become twisted, but the longitudinal field $B_{z}$ is supposed to be strong enough to prevent kink and sausage instabilities. Owing to the central bulge in the flux tube, the $\mathbf{J} \times \mathbf{B}$ force has a non-vanishing axial component, which creates flows from both foot points towards the center. The ingested plasma convects frozen-in magnetic flux from $z= \pm h$ to $z=0$, which increases the local azimuthal field $B_{\phi}$ and hence the pinch force, thus causing a reduction of the tube radius $a(0)$.

By taking the current density $J_{z}(r, z)$ to be uniform within a cross section and assuming radial pressure balance $\partial P \partial r=-J_{z} \cdot B_{\phi}$, the following expression for the total force in the $z$-direction can be derived ${ }^{5,9}$ :

$$
F_{z}=(\mathbf{J} \times \mathbf{B})_{z}-\frac{\partial P}{\partial z}=\frac{\mu_{0} I_{0}^{2}}{2 \pi^{2} a^{3}}\left(1-\frac{r^{2}}{a^{2}}\right) \frac{\partial a}{\partial z} .
$$

Obviously, $F_{z}$ and the related flows towards the center disappear for $\partial a / \partial z=0$ (i.e. for a constant tube radius along the axis). Filling the flux tube with plasma from the foot points is therefore taking place only in the collimation phase.

Furthermore, during collimation the flux surfaces are squeezed together such that the large longitudinal field $B_{z}$ becomes even larger, which would raise its energy content. Bellan ${ }^{5}$ proposed to compensate this increase of stored magnetic energy by shortening the length of the flux tube. While this is difficult to realize for the straight configuration in Fig.1, a loop with fixed foot points can reduce its length simply by shrinking. For the coronal loop simulation experiment this would mean that the apex of the arched flux tube is descending rather than ascending during the collimation phase. 


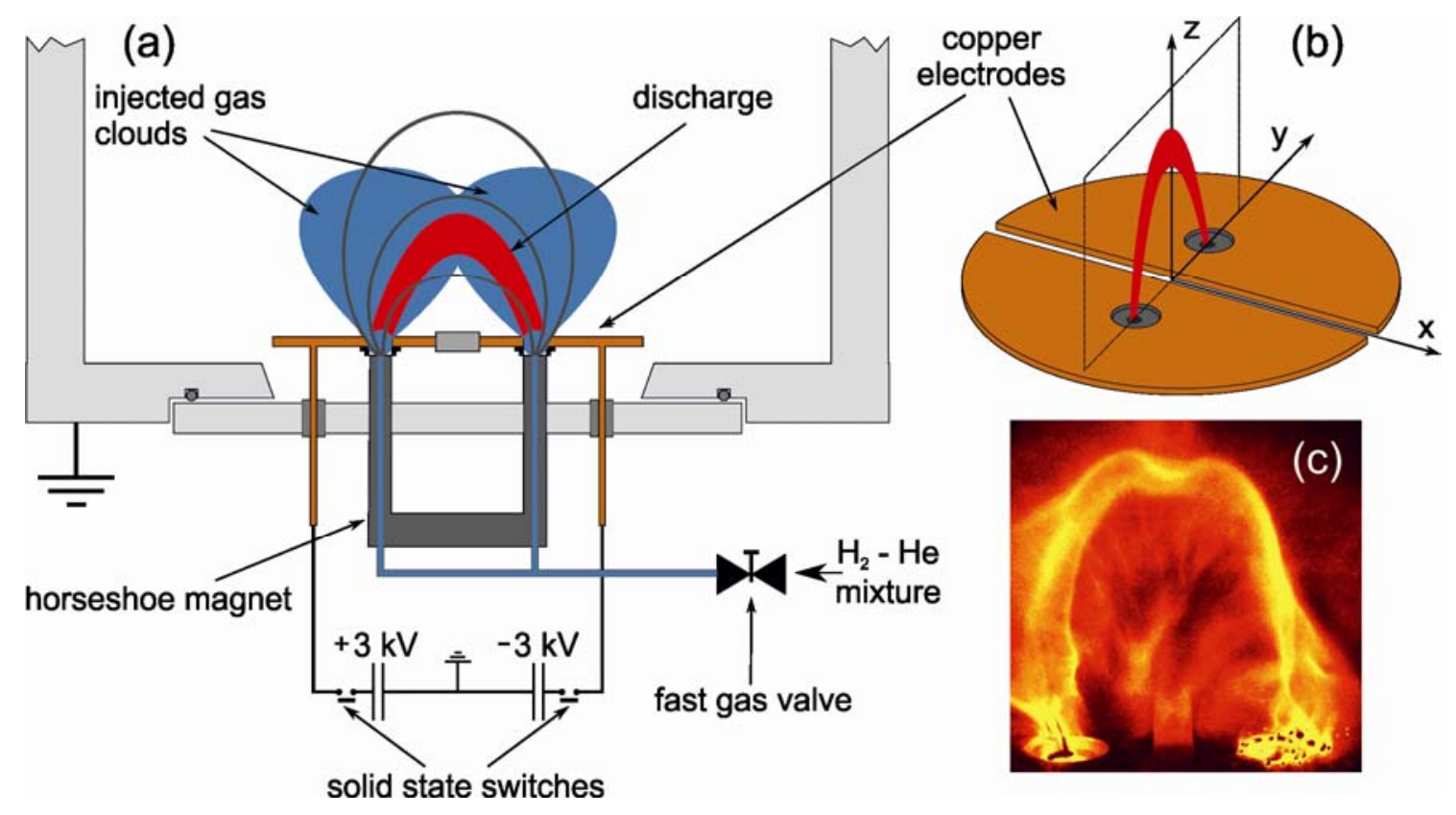

FIG. 2. (Color online) (a) Sketch of the experimental setup. (b) Semicircular electrodes with gas inlets. (c) Photograph of a discharge taken $5 \mu \mathrm{s}$ after ignition (exposure time $50 \mathrm{~ns}$ ).

\section{EXPERIMENTAL SETUP}

The FlareLab device ${ }^{10}$ at Ruhr University Bochum was designed by closely following the technical specifications and operational procedures published by Bellan and coworkers for their coronal loop simulation experiment ${ }^{3,4,11}$ at the California Institute of Technology (Caltech). Figure 2 shows a crude sketch of the setup. The discharge vessel is a stainless steel cylinder of $0.7 \mathrm{~m}$ length and $0.68 \mathrm{~m}$ diameter, which can be pumped out to a base pressure of about $10^{-4} \mathrm{~Pa}$. Below the bottom plate a horseshoe magnet is mounted, which consists of two stacks of permanent magnets and a soft-iron yoke. It produces an arch-shaped field inside the vessel with a distance between the foot points of $8 \mathrm{~cm}$ and a field strength of approximately 100 $\mathrm{mT}$ at the pole surfaces. Above the magnet two semi-circular copper electrodes are installed, which can be connected to a $1.0 \mathrm{~kJ}$ capacitor bank by means of special solid-state switches (ABB, Switzerland). The bank comprises two $120 \mu \mathrm{F}$ capacitors (symmetrically charged up to $\pm 3 \mathrm{kV}$ vs. ground) and delivers a maximum current of $35 \mathrm{kA}$ within a rise time of $14 \mu \mathrm{s}$. Gas can be supplied through the legs of the horseshoe magnet and corresponding orifices in the electrodes by means of a fast valve similar to the one described by Hansen ${ }^{11}$.

In order to run the experiment, the first step is to inject gas into the evacuated vessel by triggering the fast valve. When the two gas clouds emanating from the electrode orifices overlap (typically after a delay of 1-6 ms depending on the gas species and the length of the duct), the capacitor bank is switched across the electrodes, and 


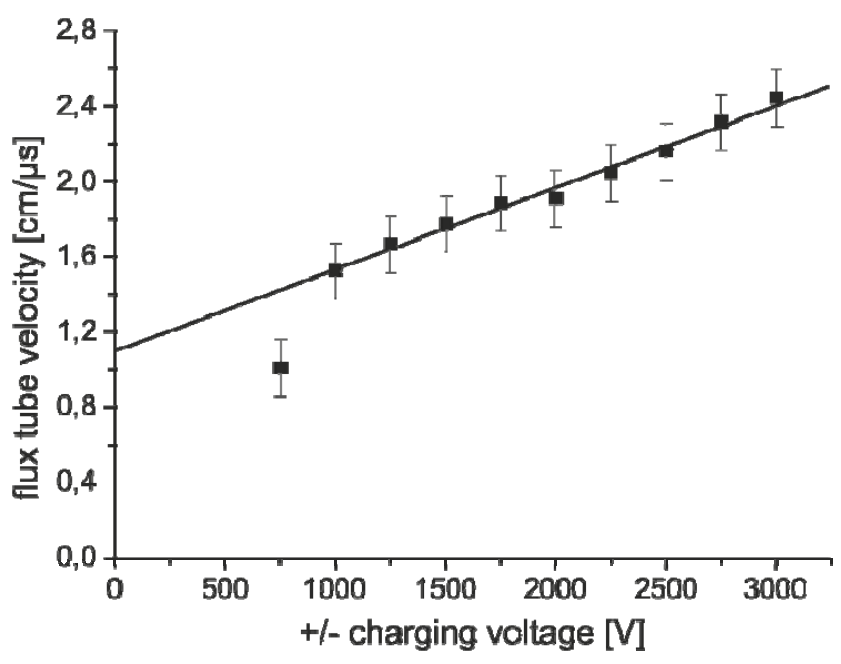

FIG. 3. Rate of ascend in the apex of the arch-shaped plasma structure as a function of the charging voltage of the main capacitor bank.

breakdown of the neutral gas occurs. Due to the overlapping clouds, the initial discharge channel has to follow an arched path instead of bridging the shortest geometrical distance, because the gas density near the electrode gap is too low to satisfy the Paschen criterion ${ }^{12}$. This has been verified by detailed measurements of the neutral gas distribution and by running discharges without horseshoe magnet to exclude the influence of a magnetic guiding field (cf. section IV.B).

While the lay-out of the electrode system including pulsed-power supply and gas feed are very similar to the original Caltech experiment (version Mark II, see Hansen ${ }^{11}$ ), differences exist with respect to the rate of current rise after breakdown and the construction of the horseshoe magnet. Owing to a lower overall inductance of the discharge circuit the maximum current in the Caltech experiment is reached after about $8 \mu \mathrm{s}$ and can be twice as high as in the FlareLab device. The archshaped guiding field in the Caltech experiment is formed by a fairly strong electromagnet and is typically $6-8$ times higher than that of the permanent magnets used in our device. However, despite these discrepancies the characteristic features of the discharges (i.e. the shape and position of the plasma in the vacuum chamber and the relevant plasma parameters) are very similar in both experiments as may be seen from two corresponding picture sequences provided as supplementary material in Ref. 6 for the Caltech experiment and in Ref. 13 for the FlareLab device.

Two intensified CCD cameras (a fast eight-frame camera to resolve the details of a developing instability and a single-frame camera providing an extended dynamic range and finer resolution) are available to follow the discharge evolution in FlareLab. In addition to these cameras the set of diagnostics comprises high-voltage probes and Rogowski coils, optical emission spectroscopy and a $\mathrm{CO}_{2}$ laser interferometer ${ }^{13}$ as well as electrostatic triple probes ${ }^{14}$ and small magnetic pick-up coils protruding into the plasma. The gross behavior of the discharges is very 
reproducible if the settings of the external parameters are kept constant. Therefore, time- and space-resolved information can be obtained even from a single probe by performing series of shots and shifting the probe in between.

We have employed the B-dot and electrostatic triple probes at variable distances from the electrode plane to measure the expansion speed and diameter of the archshaped flux tube. The results of both types of probes agree very well and correspond to the luminosity recorded by the CCD cameras. We found that the rate of ascend in the apex of the arch is surprisingly constant throughout the whole discharge evolution and has values of about $2 \mathrm{~cm} / \mu \mathrm{s}$ in a hydrogen-helium gas mixture $(20 \%$ $\mathrm{He}$ ) and $1 \mathrm{~cm} / \mu \mathrm{s}$ in argon. The exact numbers depend on the rate of current rise as shown in Fig. 3 (note that the velocity is plotted as a function of the charging voltage $\pm U_{0}$ of the capacitor bank which is proportional to $d \mathrm{l} / \mathrm{dt}$ for constant inductance of the discharge circuit). A constant rise velocity of the apex throughout the discharge evolution was also observed in the Caltech experiment by Hansen ${ }^{11}$ and by Hansen, Tripathi and Bellan ${ }^{4}$. The measured value of about $3 \mathrm{~cm} / \mu$ s was somewhat larger, presumably owing to the higher rate of current increase.

\section{EXPERIMENTAL OBSERVATIONS}

In order to examine the relevance of the proposed MHD pumping mechanism for the coronal loop simulation experiments described before, we concentrate on the temporal evolution of the flux tube morphology and of the bulk plasma density in comparison with the model predictions. Spectroscopic Doppler velocity measurements in argon discharges as reported by Tripathi, Bellan and Yun ${ }^{6}$ are less conclusive because the blue shifts of $\mathrm{Ar}^{+}$lines emitted from the legs and from the apex of the arch structure were practically the same for lines of sight normal to the electrode surfaces (i.e. for a direction of observation parallel to the loop expansion). The deduced velocities were consistent with the expansion speed which remained constant also for well collimated flux tubes where the pumping mechanism was ineffective.

\section{A. Temporal evolution of flux tube morphology}

Figure 4 shows a series of camera images of highly reproducible discharges in a hydrogen-helium gas mixture $(20 \% \mathrm{He})$ with the main capacitor bank charged to \pm 3 $\mathrm{kV}$. The labels refer to the time delays relative to the trigger pulse applied to the solid-state switches. Gas breakdown occurs approximately $1.4 \mu$ s later, hence, the first picture shows the discharge immediately after ignition. As mentioned before, the plasma luminosity coincides well with probe measurements of the width and position of the current channel and is therefore considered to be a reasonable indicator of the flux tube morphology. 


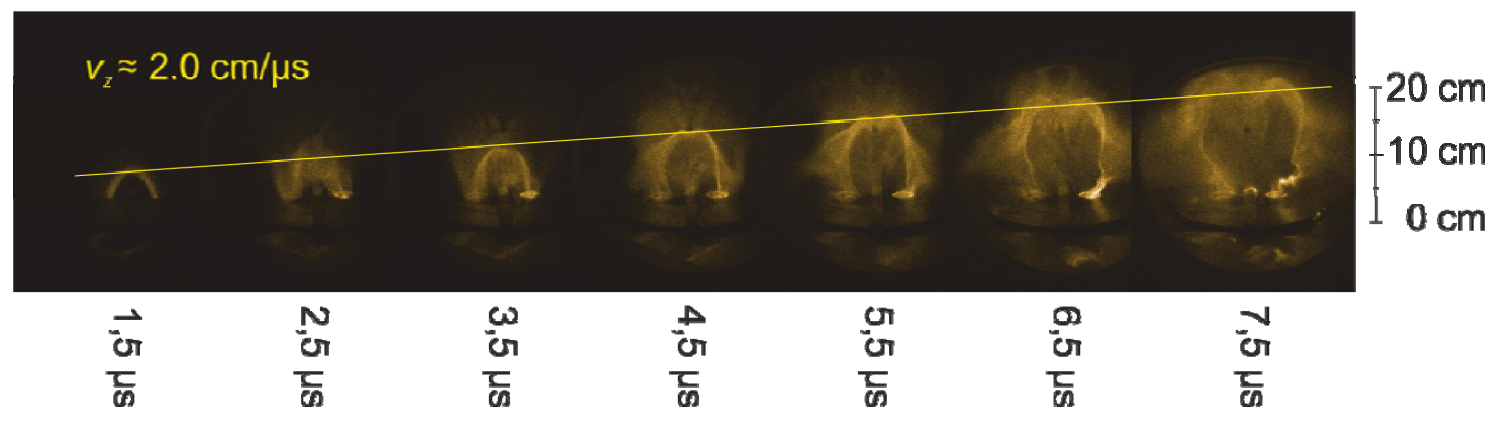

FIG. 4. (Color online) Evolution of the plasma loop for highly reproducible discharges in a $\mathrm{H}_{2}-\mathrm{He}$ gas mixture $(20 \% \mathrm{He}$; main capacitor bank charged to $\pm 3 \mathrm{kV}$; labels refer to the time delays relative to a trigger pulse activated $1.4 \mu \mathrm{s}$ before gas breakdown).

Obviously, these photographs do not support the basic model assumption of a starting configuration with a flared current profile. By contrast, the initial discharge channel is rather narrow and follows an arched path between the orifices in the electrodes. When the current starts to rise, the plasma tube appears to lose its sharp boundary and looks somewhat fuzzy. However, already less than two microseconds after gas breakdown the discharge has turned into a well-collimated flux tube with a remarkably uniform cross section along the distance between the foot points. The height of the arch is increasing at a constant speed of $v_{z} \approx 2.0 \mathrm{~cm} / \mu \mathrm{s}$. There is no indication that the apex descends rather than ascends during some initial collimation phase as predicted by the MHD pumping model.

\section{B. Temporal evolution of the plasma density}

Following Tripathi, Bellan and $\mathrm{Yun}^{6}$, the bulk plasma density in their coronal loop experiment is solely due to the MHD pumping process by ingesting particles from the electrode regions into the bulged flux tube. Consequently, the particle supply must cease when the flux tube has become collimated. On the other hand, the arched structure continues to expand and to increase its volume, which would lead to a substantial reduction of the particle density. Unfortunately, no measurements of the density evolution during the expansion phase have been published for the Caltech experiment.

In the FlareLab device we have determined the electron density in the apex of the flux loop at variable height above the electrode plane by means of a movable electrostatic triple probe ${ }^{14}$ in a series of reproducible discharges. The data were taken for the same discharge conditions as those for the images in Fig. 4. As shown in Fig.5, the maximum density measured during the passage of the flux tube across the probe is practically independent from the probe position. Hence, there must be 


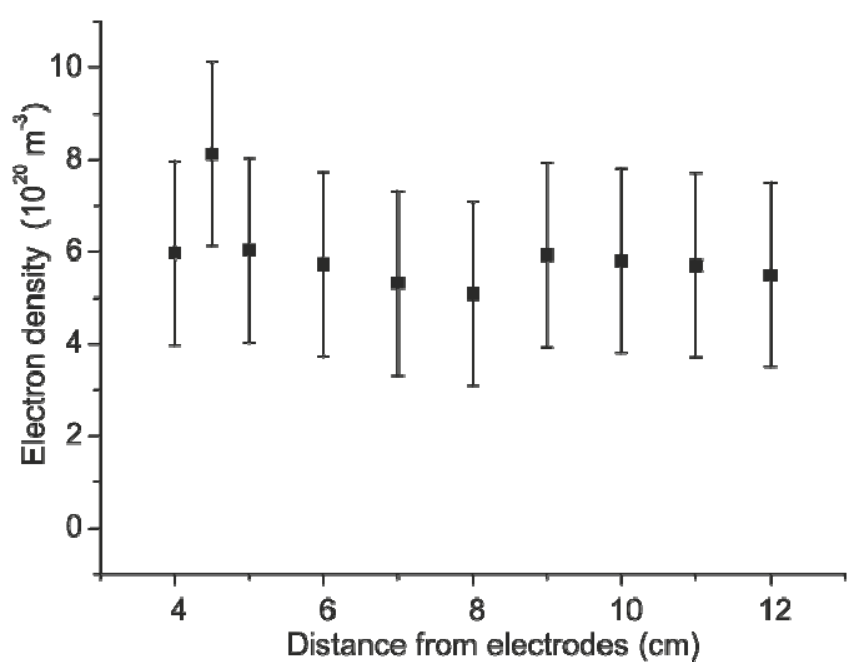

FIG. 5. Electron density in the apex of the plasma loop at variable height above the electrode plane (measurements made by means of a movable electrostatic triple probe ${ }^{14}$ in a series of reproducible discharges).

another source that allows increasing the number of particles in proportion to the volume.

The natural assumption that this source could be the injected pre-breakdown neutral gas cloud was denied by Tripathi, Bellan and Yun ${ }^{6}$. They argued that the bulk plasma in the flux loop was many orders of magnitude denser than the neutral gas existing at breakdown. This statement was based on measurements in a similar experiment by You, Yun and Bellan ${ }^{7}$ who determined the gas distribution in front of the electrodes by means of a fast ionization gauge. They found that just before breakdown the neutral gas density at the location of the discharge channel was $<10^{17} \mathrm{~m}^{-3}$. However, assuming room temperature, this value translates into a pressure of less than $4 \times 10^{-4} \mathrm{~Pa}$, which appears to be totally incompatible with the Paschen criterion ${ }^{12}$ for achieving breakdown. According to this law there exists a minimum value of the pressure-times-distance product $P d$ below which breakdown is impossible at any voltage between the electrodes. For hydrogen and argon these lower limits amount to about $1 \mathrm{~Pa} \cdot \mathrm{m}$ and $0.5 \mathrm{~Pa} \cdot \mathrm{m}$, respectively. In both gases unrealistically long paths of more than $1 \mathrm{~km}$ from one foot point to the other would be necessary if the neutral particle densities were actually as low as stated above.

In order to establish the conditions for breakdown in the FlareLab device, we mapped the distribution of the injected neutral gas without plasma as a function of space and time in the vacuum chamber by means of a home-made ionization gauge ${ }^{15}$. The proper time of discharge ignition was determined by changing the delay between firing the gas valve and sending the trigger pulse to the switches of the main capacitor bank. We found that breakdown occurred in a reproducible way 

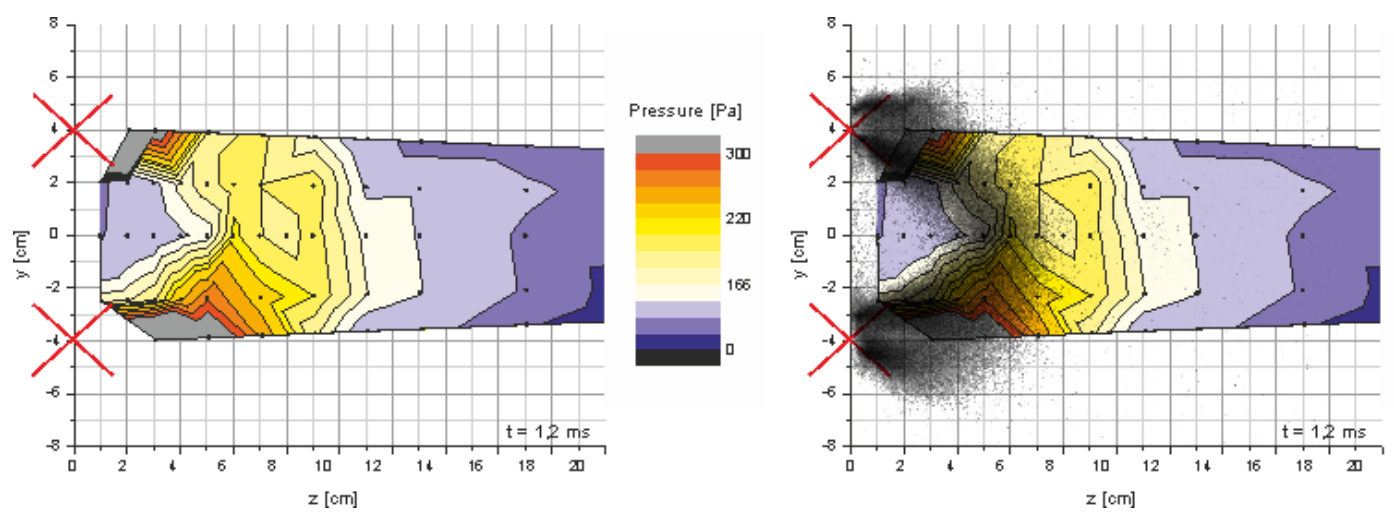

FIG. 6. (Color online) Injected gas distribution in a plane perpendicular to the electrode surfaces at the time of plasma ignition $\left(\mathrm{H}_{2}-\mathrm{He}\right.$ gas mixture with $20 \%$ of $\mathrm{He}$; crosses mark the locations of the gas orifices in the electrodes; measurements made by means of a calibrated home-made ionization gauge). Right subfigure shows a superimposed camera image of a discharge immediately after breakdown.

and with an acceptable jitter of a few nanoseconds only when the neutral gas pressure for our hydrogen-helium mixture was $150 \mathrm{~Pa}$ or more everywhere along the discharge channel. For this value the Paschen law yields a minimum distance of $>7$ $\mathrm{mm}$ to achieve ignition, which conforms well to the dimensions of the experiment. Figure 6 (left) shows the measured neutral gas distribution in a plane perpendicular to the electrode surfaces at the time of plasma ignition. The crosses mark the locations of the orifices. In Fig. 6 (right) a camera image of a plasma immediately after breakdown has been superimposed to demonstrate the importance of the background gas distribution for the initial discharge path (for taking this picture the horseshoe magnet was removed to exclude possible effects of a magnetic guiding field).

\section{PLASMA SOURCE WITHOUT BULGED MAGNETIC FIELD}

The observed discrepancies between experimental results and model predictions give rise to contesting the crucial role of the MHD pumping mechanism in the coronal loop simulation experiments under discussion. For further clarification we have modified the FlareLab device in a way that it can be operated without an initially bulged magnetic field geometry. In this configuration there is no MHD force which could ingest plasma from the electrode gas orifices and then accelerate this plasma to flow rapidly into the magnetic flux tube and to establish a fairly high density. Therefore, discharges with and without an effective pumping mechanism due to a flared magnetic field should behave quite differently, at least with respect to the achieved density and its temporal evolution. 

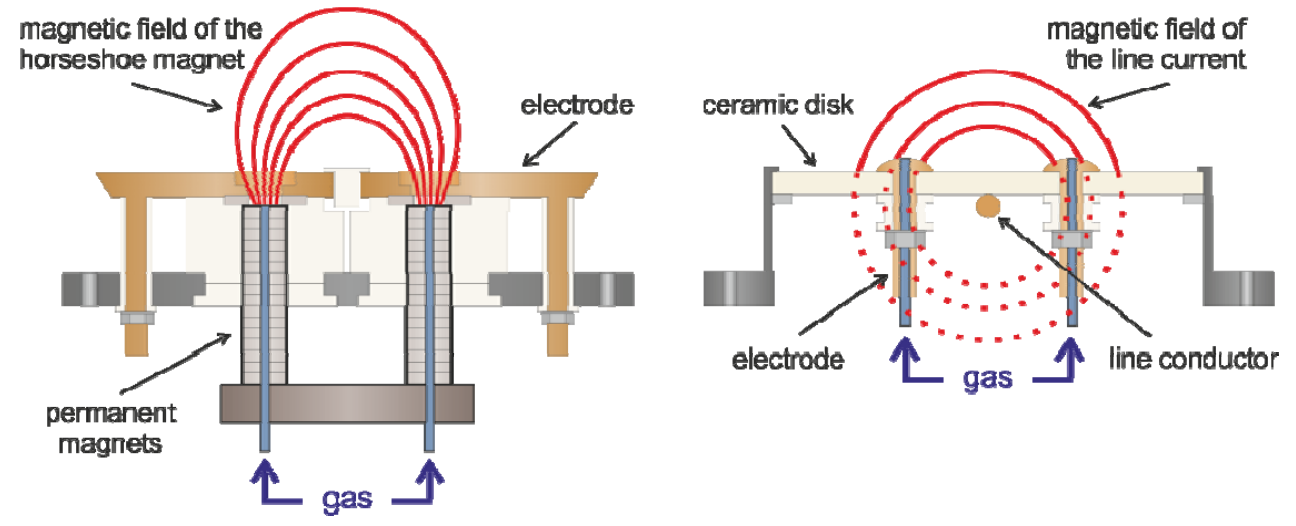

FIG. 7. (Color online) Comparison of the plasma source assemblies with horseshoe magnet (left) and line conductor (right).

As sketched in Fig. 7, the horseshoe magnet has been replaced by a strong line current which runs underneath the electrode plane and produces a set of concentric circular field lines. To reduce the effect of eddy currents, the large semi-circular electrodes have been exchanged for small disks $(2 \mathrm{~cm}$ diameter) mounted in a ceramic window for insulation and vacuum tightness. The line current of up to $25 \mathrm{kA}$ is supplied by a pulse-forming network during a plateau phase of about $12 \mu \mathrm{s}$. All other components including the main capacitor bank and the gas injection system have been left unchanged.

Most of the discharges in this modified setup were performed in argon, because the higher particle mass slows down the dynamics and makes it easier to follow the temporal changes in more detail. Usually, the device was operated with a charging voltage of $\pm 3 \mathrm{kV}$ of the main capacitor bank and a line current of $23 \mathrm{kA}$. Owing to a different zero point of the timing system (activation of the pulse-forming network for the line current), the breakdown occurs after a delay of $8 \mu \mathrm{s}$. As shown in Fig. 8 the initial discharge channel is very thin and follows exactly the magnetic field line which links the orifices in the electrodes. The subsequent evolution is very similar to the one observed in the original setup (see Fig. 4) including the kink-like deformation in the apex at a later time. As before, the arch is expanding at a constant speed, albeit with a smaller rate of ascend of $v_{z} \approx 0.9 \mathrm{~cm} / \mu \mathrm{s}$.

In order to determine the electron density in the apex of the flux loop with higher precision, the movable triple probe was replaced by a $\mathrm{CO}_{2}$ laser interferometer whose probing beam could be shifted inside the vessel by means of a vacuumcompatible translation stage ${ }^{16,17}$. Results are shown in Fig. 9, where the interferometric phase shift in units of line-integrated electron density is plotted in color code as a function of time and position along the central line perpendicular to the electrode plane (corresponding to the $z$ axis in Fig. 2). The constant expansion 


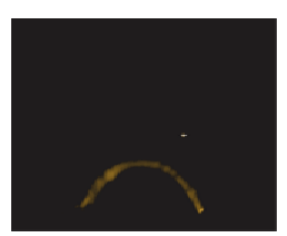

$\overbrace{\infty}^{\infty}$

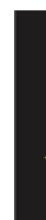
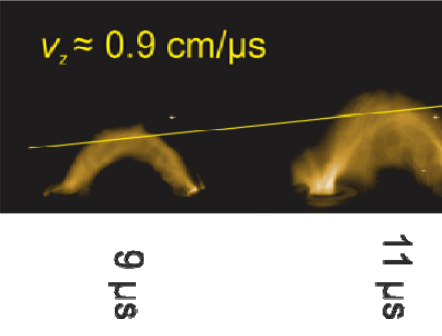

FIG. 8. (Color online) Evolution of the plasma loop for highly reproducible discharges in the modified plasma source (argon gas; main capacitor bank charged to $\pm 3 \mathrm{kV}$; circular magnetic field lines produced by a $23 \mathrm{kA}$ line current; labels refer to the time delays relative to a trigger pulse activated 8 $\mu$ s before gas breakdown).

velocity of the arch and the slowly increasing width of the density profile agree very well with the camera pictures of fig. 8 (note that the apparent periodic modulation in Fig. 9 is an artifact caused by the plot program). Absolute numbers of the electron density, which can be deduced from this graph, are of the same order of magnitude as those measured in the original setup. Obviously, the pump mechanism is not required to achieve a plasma bulk density of more than $10^{21} \mathrm{~m}^{-3}$, because the injected neutral gas cloud can already provide sufficiently many plasma particles by ionization.
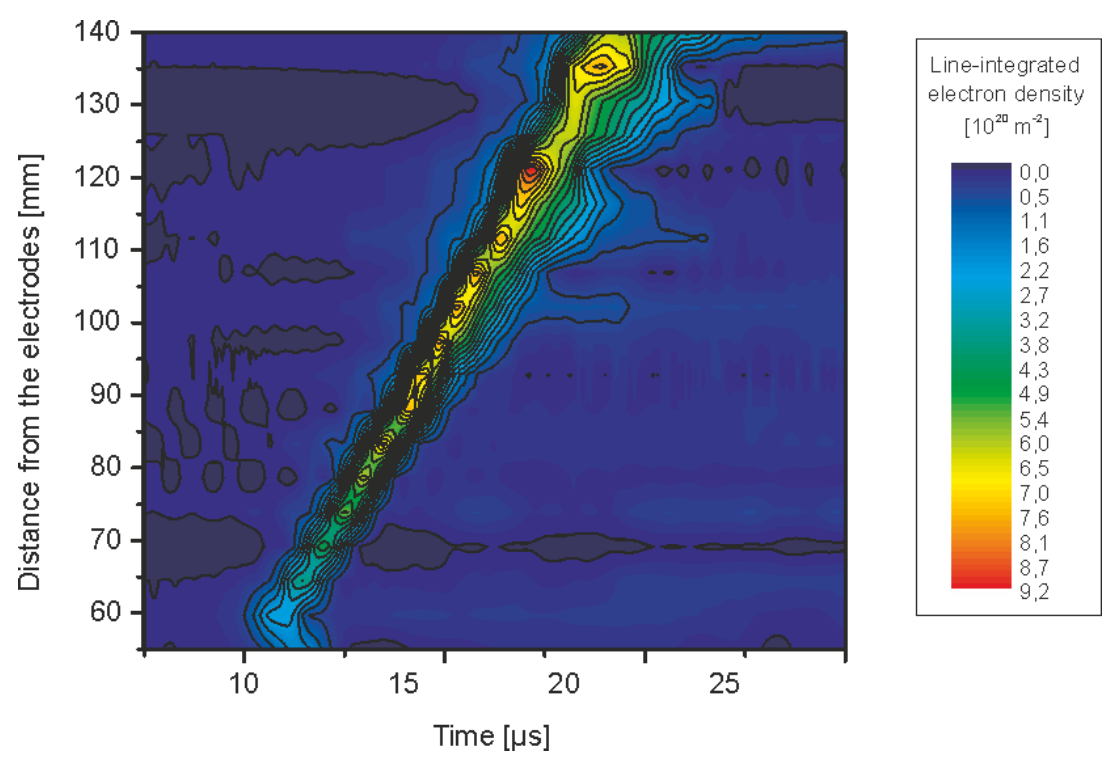

FIG. 9. (Color online) Interferometric phase shift in units of line-integrated electron density shown in color code as a function of time and position along the central line perpendicular to the electrode plane (corresponding to the $z$ axis in Fig. 2). 


\section{SUMMARY}

In the present paper we contrast Bellan's MHD pumping model ${ }^{5}$, which was developed to explain the general behavior of initially bulged, current-carrying magnetic flux tubes, with measurements and observations in an appropriate laboratory experiment. The device was built in close analogy to a Caltech experiment ${ }^{11}$ for the simulation of solar coronal loops. Whilst the Caltech group interpreted their results as being in accordance with the pumping model, we found significant discrepancies with respect to the predicted temporal evolution of the flux tube morphology and the plasma density. In particular, we have not been able to verify an increase of the plasma bulk density by many orders of magnitude over the local neutral gas density at the time of breakdown as stated in Ref. 6.

In order to compare the formation and temporal evolution of the plasma channel with and without the assumed pumping mechanism, we have replaced the initially bulged magnetic field geometry by concentric circular field lines surrounding a strong electrical current. Since we could not observe significant differences between both magnetic field configurations, we conclude that the proposed MHD pumping mechanism does not play a role in the investigated solar coronal loop simulation experiments. With this statement we do not want to dispute the basic ideas underlying the model, but we believe that the given experimental conditions (especially in the early phase after breakdown) do not satisfy the prerequisites of ideal MHD sufficiently well to warrant the applied simplifications.

\section{ACKNOWLEDGMENTS}

The support of this work by the Forschergruppe 1048 of the Deutsche Forschungsgemeinschaft, by the Research Department 'Plasmas with Complex Interactions' of the Ruhr University Bochum, and by the Ruhr University Research School is gratefully acknowledged.

\section{REFERENCES}

${ }^{1}$ Coronal Mass Ejections, edited by N. Crooker, J. A. Joselyn, and J. Feynman (American Geophysical Union Monograph, Washington, DC, 1997).

${ }^{2}$ T. Török and B. Kliem, Astrophys. J. 630, L97 (2005).

${ }^{3}$ P.M. Bellan and J. F. Hansen, Phys. Plasmas 5, 1991 (1998).

${ }^{4}$ J.F. Hansen, S.K.P. Tripathi and P.M. Bellan, Phys. Plasmas 11, 3177 (2004). 
${ }^{5}$ P.M. Bellan, Phys. Plasmas 10, 1999 (2003).

${ }^{6}$ S. K. P. Tripathi, P. M. Bellan, and G. S. Yun, Phys. Rev. Lett. 98, 135002 (2007).

${ }^{7}$ S. You, G. S. Yun, and P. M. Bellan, Phys. Rev. Lett 95, 045002 (2005).

${ }^{8}$ G. S. Yun, S. You, and P. M. Bellan, Nucl. Fusion 47, 181 (2007).

${ }^{9}$ G. S. Yun and P. M. Bellan, Phys. Plasmas 17, 062108 (2010).

${ }^{10} \mathrm{H}$. Soltwisch, P. Kempkes, F. Mackel, H. Stein, J. Tenfelde, L. Arnold, J. Dreher, and R. Grauer, Plasma Phys. Control. Fusion 52, 124030 (2010).

11J. F. Hansen, Ph.D. Thesis, California Institute of Technology, Pasadena, 2001.

${ }^{12}$ P. M. Bellan, Spheromaks (Imperial College Press, London, 2000).

${ }^{13}$ P. Kempkes, F. Mackel, H. Stein, J. Tenfelde, and H. Soltwisch, Meas. Sci. Technol. 21, 125701 (2010).

${ }^{14}$ F. Mackel, P. Kempkes, H. Stein, J. Tenfelde, and H. Soltwisch, Meas. Sci. Technol. 22, 055705 (2011).

${ }^{15}$ T. Tacke, Diploma Thesis, Ruhr University, Bochum, 2011.

${ }^{16}$ S. Ridder, DiplomaThesis, Ruhr University, Bochum, 2011.

${ }^{17}$ F. Mackel, P. Kempkes, J. Tenfelde, S. Ridder, and H. Soltwisch, "Triple probe measurements benchmarked by interferometry in a pulsed-power plasma", accepted for publication in Contrib. Plasma Phys. 